\title{
Techniques of Reducing Switching Artifacts in Chopper Amplifiers
}

\author{
Yoshinori KUSUDA $^{\dagger \text { a) }}$, Nonmember
}

SUMMARY Chopping technique up-modulates amplifier's offset and low-frequency noise up to its switching frequency, and therefore can achieve low offset and low temperature drift. On the other hand, it generates unwanted AC and DC errors due to its switching artifacts such as up-modulated ripple and glitches. This paper summarizes various circuit techniques of reducing such switching artifacts, and then discusses the advantages and disadvantages of each technique. The comparison shows that newer designs with advanced circuit techniques can achieve lower DC and AC errors with higher chopping frequency.

key words: amplifier, chopping, switching artifact, charge injection

\section{Introduction}

Chopping is a circuit technique that involves up-modulating amplifier offset and low frequency noise to higher frequencies, and which therefore can achieve microvolt-level offset with very low temperature drift [1]. On the other hand, it causes switching artifacts such as AC ripple and glitches at the amplifier's output, which usually need to be attenuated by low-pass filtering, thus decreasing the usable signal bandwidth [2]. This paper introduces advanced circuit techniques to reduce such switching artifacts over the past years as well as their advantages and disadvantages.

\subsection{Principle of Chopping and Up-Modulated Ripple}

Figure 1 (a) shows a circuit diagram of a chopper operational amplifier (op-amp) in a closed-loop. The chopper opamp consists of an input chopper $\mathrm{CH}_{\mathrm{IN}}$, an input transconductor $G_{m l}$ with its undesired offset $V_{o s l}$, an output chopper $\mathrm{CH}_{\mathrm{OUT}}$, and an output transconductor $G_{m 2}$. The choppers $\mathrm{CH}_{\mathrm{IN}}$ and $\mathrm{CH}_{\text {OUT }}$ perform the modulation by two phase clocks $\Phi_{\mathrm{CH}}$ and $\Phi_{\mathrm{CHINV}}$ operating at a chopping frequency $f_{C H}$ as shown in a timing diagram Fig. 1 (b). Provided that $f_{C H}$ is well below the closed-loop bandwidth around the opamp, the amplitude of up-modulated ripple $V_{\text {out,rip }}$ is given by:

$$
\begin{aligned}
& V_{\text {out }, \text { rip }}=A_{C L} V_{\text {os } 1} \\
& A_{C L}=\left(1+R_{F} / R_{G}\right),
\end{aligned}
$$

where $A_{C L}$ is the closed-loop gain determined by external resistors $R_{G}$ and $R_{F}$.

Manuscript received November 12, 2019.

Manuscript publicized April 9, 2020.

$\dagger$ The author is with Analog Devices, Inc., Santa Clara, CA 95054 U.S.A.

a) E-mail: yoshinori.kusuda@ analog.com

DOI: 10.1587/transele.2019CTI0001

\subsection{Glitches}

In addition to the ripple, charge injections of input chopper switches introduce glitches as shown in Fig. 2. First, $\Phi_{\mathrm{CH}}$ drops, injecting negative charge on the differential input pins. Soon thereafter, $\Phi_{\mathrm{CHINV}}$ rises, injecting almost the same amount of charges but with positive polarity. Ideally, these negative and positive charges cancel out instantaneously at each input pin, if the transitions of the two clocks occur at the exact same time. In practice, however, there must be some dead time between the two clocks, in order not to short the differential input pins with the switches. The glitch amplitude depends on the source impedances, the dead time, and the amount of charge injection. Similarly, after a half chopping period, $\Phi_{\mathrm{CHINV}}$ drops and $\Phi_{\mathrm{CH}}$ rises, resulting in another glitch.

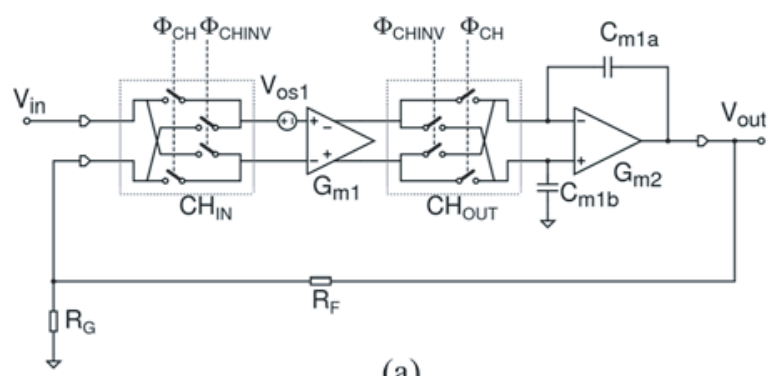

(a)

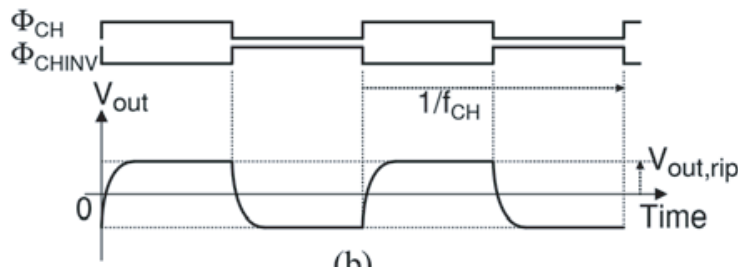

(b)

Fig. 1 Chopper op-amp in a closed-loop: (a) circuit diagram; (b) chopper clock and output ripple waveform.

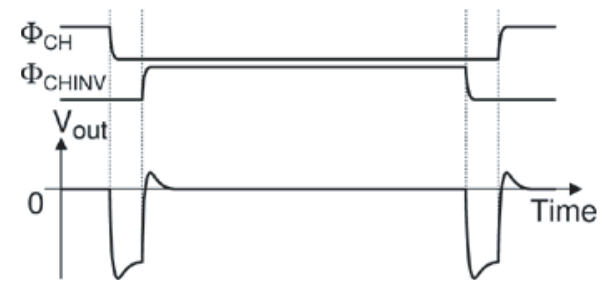

Fig. 2 Output glitch due to input charge injection. 
Figure 3 shows the output noise power spectral density (PSD) of a chopper op-amp, taking both the ripple and the glitches into account. The PSD increases at the odd harmonics of the chopping frequency $f_{C H}$ due to the up-modulated ripple, and at the even harmonics of $f_{C H}$ due to the glitches. These noise spectra peaks usually need to be attenuated by a post low-pass filter, which increases cost and limits the usable signal bandwidth.

\subsection{Input Bias Current and Residual Offset}

Charge injection mismatch causes DC errors such as residual offset and input bias current [2], [3]. Close inspection shows that only the charge injection mismatch in the signal path between the two choppers is down-modulated, and thus contributes to the DC errors. As shown in Fig. 4 (a), $\mathrm{CH}_{\mathrm{IN}}$ consists of four switches $\mathrm{S}_{1-4}$, which inject charges $q_{1-4}$ during every clock transition.

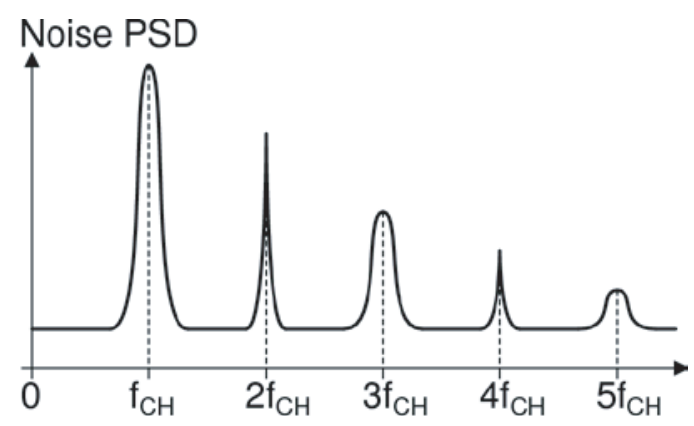

Fig. 3 Output noise PSD of a chopper op-amp.

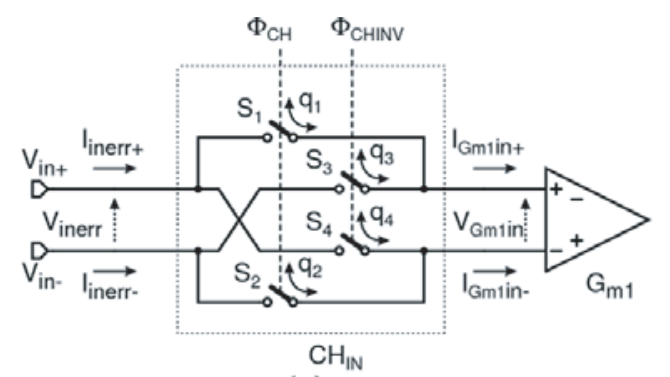

(a)

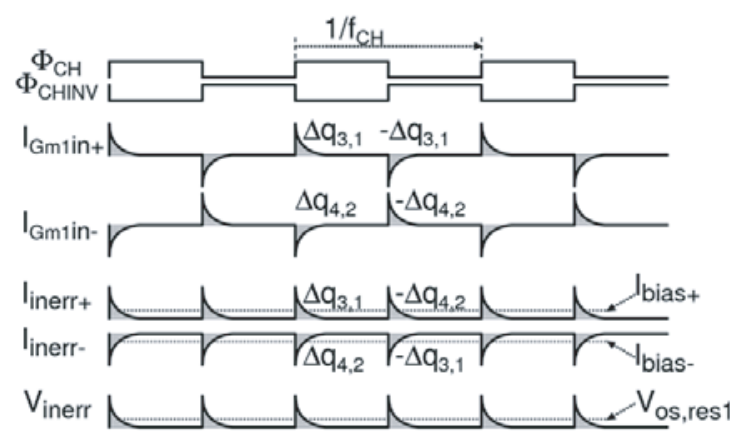

(b)

Fig. 4 Input bias current and residual offset due to input chopping: (a) circuit diagram; (b) transient waveform.
As shown in Fig. 4 (b), charge injection mismatches $\Delta q_{3,1}=q_{3}-q_{1}$ and $\Delta q_{4,2}=q_{4}-q_{2}$ are down-modulated so that the transient input currents $I_{\text {inerr }+}$ and $I_{\text {inerr }}$ include the DC bias components $I_{\text {bias }+}$ and $I_{\text {bias }}$ given by:

$$
\begin{aligned}
I_{\text {bias }} & =f_{C H}\left(\Delta q_{3,1}-\Delta q_{4,2}\right) \\
I_{\text {bias- }} & =-f_{C H}\left(\Delta q_{3,1}-\Delta q_{4,2}\right) .
\end{aligned}
$$

Moreover, in conjunction with the on-resistance of each switch $R_{o n}$ and the source resistances driving the op-amp $\left(R_{S_{+}}\right.$and $\left.R_{S_{-}}\right)$, these input bias currents cause a residual input offset voltage $V_{o s, r e s}$ equal to:

$$
V_{o s, r e s}=f_{C H}\left(\Delta q_{3,1}-\Delta q_{4,2}\right)\left(2 R_{o n}+R_{S+}+R_{S-}\right) \text {. }
$$

\subsection{Summery of AC and DC Errors Due to Switching Ar-} tifacts

Many op-amp users prefer higher chopping frequency $f_{C H}$ to locate the noise spectra peaks at higher frequencies and to extend the usable signal bandwidth. However, this increases the residual offset and input bias current. Those DC errors will also increase proportional to the amount of charge injection mismatch when wider input switches are used to reduce the on-resistances, hence the thermal noise. As discussed in later sections, researchers have invented various circuit techniques to reduce such $\mathrm{AC}$ and DC errors in chopper op-amps.

\section{Ripple Reduction Techniques}

\subsection{Multi-Path Chopper Offset Stabilization}

To attenuate the ripple amplitude, Witte proposed multi-path chopper offset stabilized op-amp employing active filter in 2006 [4].

As shown in the circuit diagram in Fig. 5, the proposed op-amp consists of a low frequency path (LFP) and a high frequency path (HFP) in parallel. The LFP further consists of the input transconductor $G_{m l}$ and the subsequent three transconductors $G_{m 2-4}$, whereas the HFP consists of the other input transconductor $G_{m 5}$ and shares $G_{m 4}$ with the LFP. The LFP dominates the low frequency performance of the overall op-amp, and $G_{m l}$ is chopped to achieve low offset. The up-modulated input offset $V_{o s I}$ is filtered by two

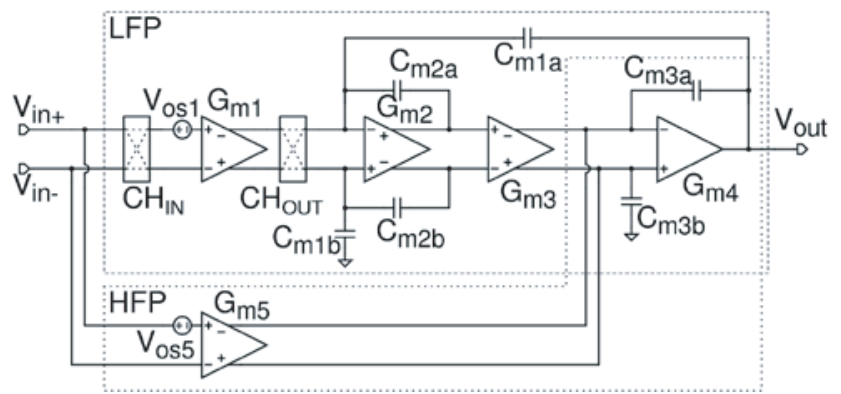

Fig. 5 Multi-path chopper offset stabilized op-amp. 


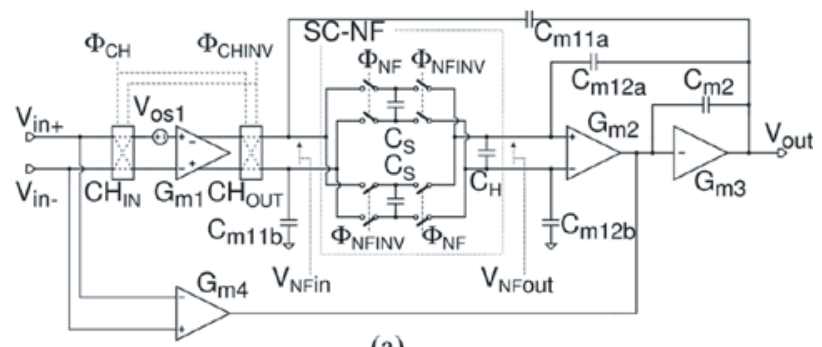

(a)

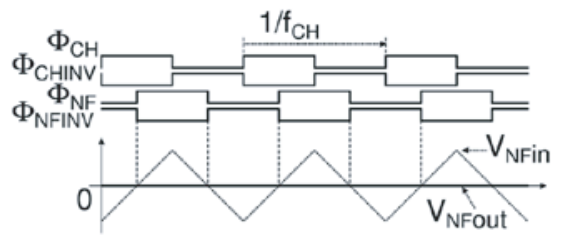

(b)

Fig. 6 Chopper op-amp employing a SC notch-filter: (a) circuit diagram; (b) timing diagram.

integrators built around $G_{m 2}$ and $G_{m 4}$. The output ripple amplitude $V_{\text {out }, \text { rip }}$ is then given by:

$$
V_{\text {out }, \text { rip }}=\frac{G_{m 1}}{2 f_{C H} C_{m 2 a, b}} \cdot \frac{G_{m 3}}{G_{m 5}} A_{C L} V_{o s 1},
$$

where $A_{C L}$ is the closed-loop gain set by the external resistors. $V_{\text {out,rip }}$ can be reduced by adjusting parameters in the LFP such as $G_{m l}, G_{m 3}$, and $C_{m 2 a, b}$. On the other hand, the unity gain bandwidth is set by the HFP and is equal to $G_{m 5} /\left(2 \pi C_{m 3 a, b}\right)$. The offset of the HFP $\left(V_{o s}\right)$ is offset stabilized by the LFP. However, in conjunction with the finite voltage gain of the LFP, it produces a residual offset $V_{o s, r e s}$ given by:

$$
V_{o s, r e s}=\frac{A_{v 5}}{A_{v 1} A_{v 2} A_{v 3}} V_{o s 5},
$$

where $A_{v 1,2,3,5}$ are the DC voltage gains of $G_{m 1,2,3,5}$, respectively. One drawback is that this technique requires additional transconductors and capacitors to realize multiple integrators.

\subsection{Switched Capacitor Notch Filter}

Since the phase of the ripple is aligned with the chopping clocks, a switched capacitor notch filter (SC-NF) can be implemented with clocks synchronized with the chopping.

Figure 6 (a) shows a block diagram of the op-amp proposed by Burt in 2006 [5]. This again consists of a LFP and a HFP, where the LFP includes a SC-NF following the chopped input transconductor $G_{m l}$. Figure $6(\mathrm{~b})$ shows the timing diagram of the chopping $\left(\Phi_{\mathrm{CH}}\right.$ and $\left.\Phi_{\mathrm{CHINV}}\right)$ and the SC-NF ( $\Phi_{\mathrm{NF}}$ and $\left.\Phi_{\mathrm{NFINV}}\right)$, as well as the input and output voltages of the SC-NF $\left(V_{N F i n}\right.$ and $\left.V_{N F o u t}\right)$. First, $V_{N F i n}$ contains a triangular ripple due to the up-modulated output current of $G_{m l}$. However, since $\Phi_{\mathrm{NF}}$ and $\Phi_{\mathrm{NFINV}}$ operate at the same frequency as $\Phi_{\mathrm{CH}}$ and $\Phi_{\mathrm{CHINV}}$ but with a 90 -degree phase difference, the SC-NF samples the triangular ripple

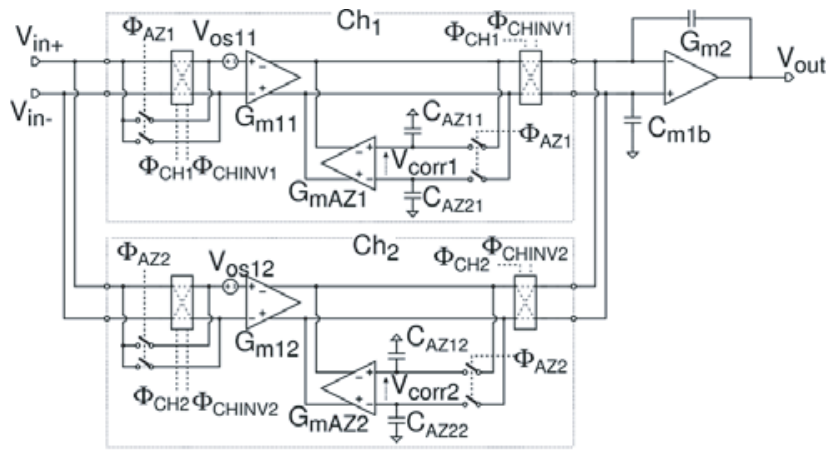

(a)

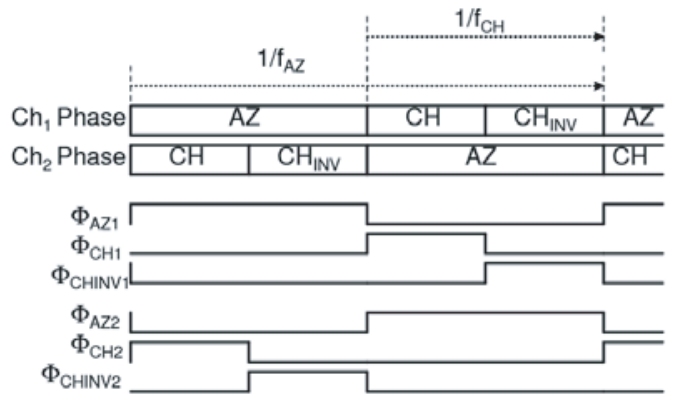

(b)

Fig. 7 A ping-pong auto-zero and chopper op-amp: (a) circuit diagram; (b) timing diagram.

whenever the amplitude becomes zero, and thus results in the ripple free output voltage $V_{\text {NFout }}$. This SC-NF adds a 90degree phase delay to the signal at the chopping frequency, so that the HFP is required to bypass this phase delay and to stabilize the overall op-amp.

This filter does not consume quiescent current, and thus is more power efficient than active filters so that the proposed op-amp only consumes $17 \mu \mathrm{A}$ of current. However, there are some drawbacks. First, clock skew and sampling capacitor mismatch result in residual ripple. Second, the sampled noise onto $C_{S}$ can contribute to the overall in-band noise PSD. Third, $G_{m l}$ must have a wide output range to tolerate the maximum ripple amplitude, since the ripple still exists there. This problem becomes more severe in low noise designs, because the ripple amplitude at $V_{N F i n}$ is proportional to $G_{m l} / C_{S}$.

\subsection{Combination with Auto-Zeroing}

Instead of filtering, the ripple amplitude can be reduced by reducing the initial offset before being up-modulated. In 2002, Tang proposed combining auto-zeroing and chopping the block diagram of which is shown in Fig. 7 (a) [6].

To achieve continuous-time amplification, it pingpongs two input channels $\mathrm{Ch} 1$ and $\mathrm{Ch} 2$, followed by an output stage $G_{m 2}$. As shown in the timing diagram Fig. 7 (b), each channel is driven by three clocks $\Phi_{\mathrm{AZ1} 1,2}, \Phi_{\mathrm{CH} 1,2}$, and $\Phi_{\mathrm{CHINV} 1,2}$, to define auto-zeroing (AZ), chopping $(\mathrm{CH})$, and inverting-chop $\left(\mathrm{CH}_{\mathrm{INV}}\right)$ phases. When one channel is in the 
$\mathrm{AZ}$ phase, the other goes through $\mathrm{CH}$ and $\mathrm{CH}_{\mathrm{INV}}$ phases. At the end of each $\mathrm{AZ}$ phase, the offset correction signals $V_{\text {corr } 1,2}$ are sampled on capacitors $C_{A Z 11,21,12,22}$ to cancel each initial offset $V_{o s 11,12}$. The resulting output ripple is reduced by a factor of the auto-zeroing loop, which can be usually higher than $80 \mathrm{~dB}$ by utilizing cascode topology. The chopping frequency $f_{C H}$ is set at twice the auto-zeroing frequency $f_{A Z}$.

Figure 8 (a), (b), and (c) show the expected noise PSDs of before auto-zeroing or chopping, after the auto-zeroing, and after the auto-zeroing and the chopping, respectively. The auto-zeroing almost eliminates $1 / f$ noise, but introduces increased in-band noise PSD due to the broadband noise PSD folding [1]. This increased noise PSD lies in be-

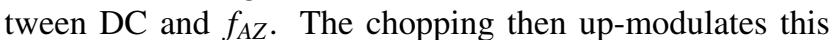
high noise PSD up to $f_{C H}$, to obtain an in-band noise PSD that is only slightly higher than the initial thermal noise floor.

This op-amp achieves a $3 \mu \mathrm{V}$ offset and a $20 \mathrm{nV} / \sqrt{\mathrm{Hz}}$ in-band noise PSD, while consuming $800 \mu \mathrm{A}$ of current. One drawback is the additional current consumption and die area for one extra input channel.

\subsection{Auto-Correction Feedback}

In 2010, Kusuda proposed auto-correction feedback (ACFB) to reduce the initial offset, hence the ripple, without requiring auto-zeroing [7], [8].

Figure 9 shows the circuit diagram of the proposed opamp which consists of a low frequency path (LFP) by three transconductors $G_{m 1-3}$, and a high frequency path (HFP) by transconductors $G_{m 4}$ and $G_{m 3}$. The initial offset $V_{o s l}$ is upmodulated and results in ripples at the output $V_{\text {out }}$ and an

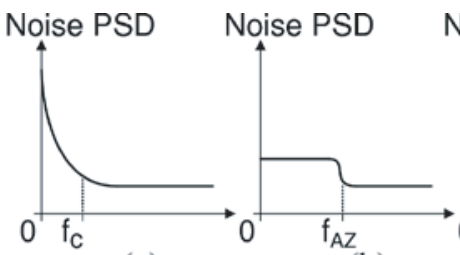

(a)

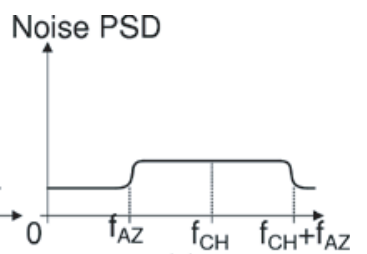

(c)
Fig. 8 Noise PSDs (a) without auto-zeroing or chopping, (b) after autozeroing, and (c) after auto-zeroing and chopping.

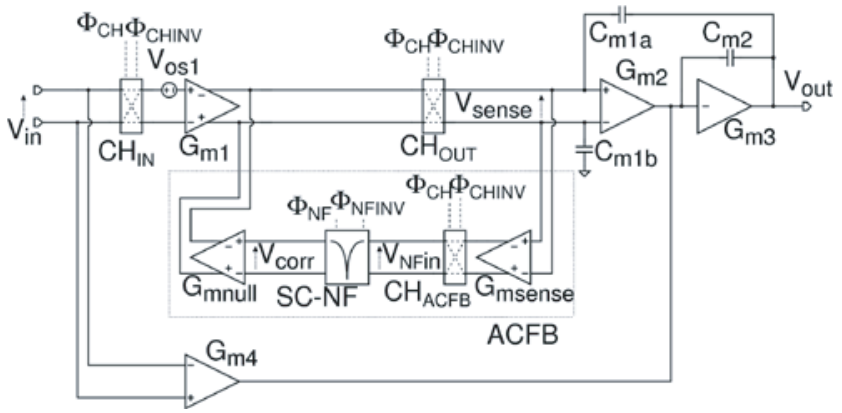

Fig. 9 Chopper op-amp employing auto-correction feedback. internal node $V_{\text {sense }}$. The ACFB senses the ripple at $V_{\text {sense }}$, down-modulates it by $\mathrm{CH}_{\mathrm{ACFB}}$, and generates an offset correction signal $V_{\text {corr }}$. Based on $V_{\text {corr }}$ and the feedback of the ACFB, an offset nulling transconductor $G_{\text {mnull }}$ cancels $V_{o s}$ to reduce the output ripple amplitude. A switched capacitor notch filter (SC-NF) is controlled by the same clock timings as the one in Fig. 6 (b) $-\Phi_{\mathrm{NF}}$ and $\Phi_{\mathrm{NFINV}}$ operate at the same frequency as the chopping clocks $\Phi_{\mathrm{CH}}$ and $\Phi_{\mathrm{CHINV}}$ but with a 90-degree phase difference. The desired input signal $V_{\text {in }}$ results in a triangular ripple at $V_{N F, I N}$ after being modulated by $\mathrm{CH}_{\mathrm{IN}}, \mathrm{CH}_{\mathrm{OUT}}$, and $\mathrm{CH}_{\mathrm{ACFB}}$. This ripple is filtered by the SC-NF, such that the ACFB does not affect $V_{i n}$.

The op-amp presented in [8] achieves $0.5 \mu \mathrm{V}$ maximum offset and $5.6 \mathrm{nV} / \sqrt{\mathrm{Hz}}$ noise PSD while consuming $1.4 \mathrm{~mA}$ of current. Compared to the implementation in [6], which uses a SC-NF in the main signal path, the ACFB requires two extra transconductors to sense the ripple and to cancel the initial offset, although they only consume $6 \%$ of the total current. On the other hand, when desired, the ACFB offers flexibility in choosing a relatively low chopping frequency, which reduces the input bias current. Moreover, the sampled noise in the SC-NF is up-modulated and thus does not contribute to the resulting in-band noise PSD.

\section{Glitch Reduction Techniques}

\subsection{Adaptive Clock Boosting Technique}

As explained in the Sects. 1.2 and 1.3, charge injections of input chopper switches cause transient output glitches, and the charge injection mismatch leads to a residual offset and input bias current. On the other hand, the switches must be made sufficiently wide to reduce the on-resistance and the thermal noise.

This requires a trade-off among the in-band noise PSD, the output glitches, and the residual offset in a chopper opamp design. Moreover, the on-resistance of a conventional CMOS switch changes over the input common-mode voltage $\left(V_{C M}\right)$ and the supply voltage $\left(V_{S Y}\right)$ due to the change of its overdrive voltage.

Figure 10 (a) and (b) shows the on-conductances of an

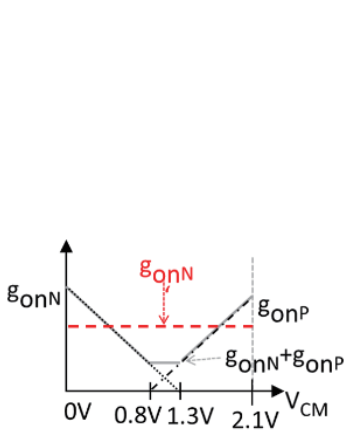

(a)

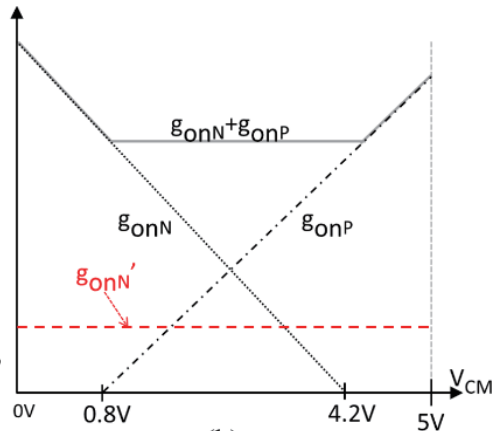

(b)
Fig. 10 On conductances of a conventional CMOS switch gonN+gonP and a NMOS switch with a constant overdrive voltage gonN' (a) with $\mathrm{VSY}=2.1 \mathrm{~V}$ and $(\mathrm{b}) \mathrm{VSY}=5 \mathrm{~V}$ 


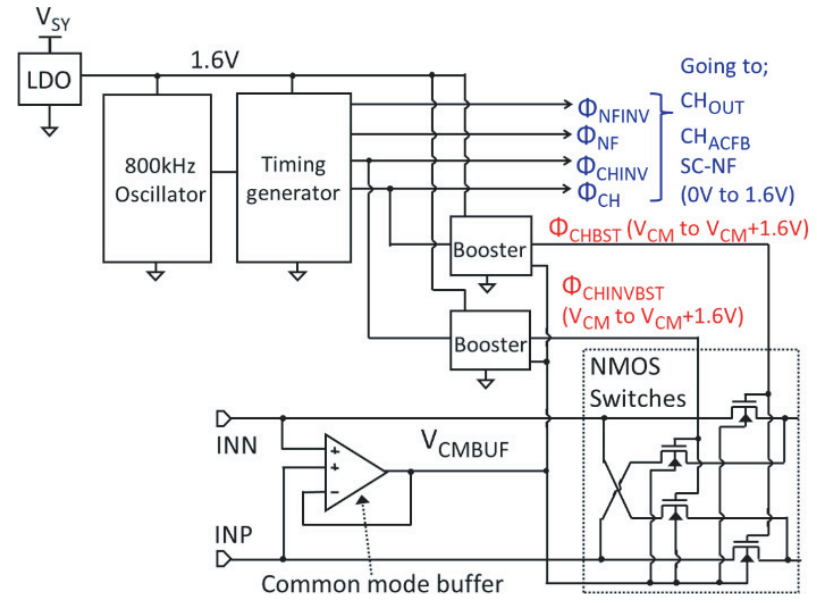

Fig. 11 Input chopping circuit and clock generator employing an adaptive clock boosting technique.

NMOS $g_{\text {on }}$, a PMOS $g_{\text {onP }}$, and a CMOS switch $g_{\text {onN }}+g_{\text {on } P}$ over a rail-to-rail $V_{C M}$ range with $V_{S Y}=2.1$ and $5.0 \mathrm{~V}$, respectively. The PMOS is typically sized three times as wide as the NMOS to compensate for the lower mobility of holes compared to electrons. The on-conductance $g_{\text {onN }}+g_{\text {onP }}$ is lowest, when $V_{S Y}$ is the lowest and $V_{C M}$ is in the middle of $V_{S Y}$. Thus, the switches must be made sufficiently wide to achieve the targeted noise PSD for this voltage condition. Such wide switches are oversized for the other voltage conditions, and thus generate more charge injection. This increases transient glitches and residual offset, and degrades the CMRR and the PSRR. This problem can be solved by maintaining the overdrive voltage of an NMOS switch constant and independent of changes in $V_{S Y}$ and $V_{C M}$, so that its on-conductance $g_{\text {onN }}{ }^{\prime}$ remains constant as shown in Fig. 10 (a) and (b).

Figure 11 presents the proposed NMOS input chopper $\mathrm{CH}_{\text {IN }}$ as well as the clock boosters to maintain the overdrive voltage constant. This circuit is used in the chopper op-amp shown in Fig. 9 [8]. The clock generator consists of an $800 \mathrm{kHz}$ oscillator and a timing generator based on D-latches, resulting in two sets of $200 \mathrm{kHz}$ complementary clocks with a 90 -degree phase shift $\Phi_{\mathrm{CH}}, \Phi_{\mathrm{CHINV}}$ and $\Phi_{\mathrm{NF}}$, $\Phi_{\text {NFINV }}$. Supplied by a $1.6 V$ LDO, those clocks have a fixed $1.6 \mathrm{~V}$ amplitude to drive NMOS switches other than input switches, i.e. $\mathrm{CH}_{\mathrm{OUT}}, \mathrm{CH}_{\mathrm{ACFB}}$, and the SC-NF.

Based on $\Phi_{\mathrm{CH}}$ and $\Phi_{\mathrm{CHINV}}$, two identical adaptive clock boosters generate the boosted output clocks $\Phi_{\text {CHBST }}$ and $\Phi_{\text {CHINVBST }}$ by utilizing the 1.6V LDO output and a buffered input common-mode voltage $V_{C M B U F}$ from a commonmode buffer. In each phase, one adaptive clock booster drives two NMOS switches to $V_{C M B U F}+1.6 \mathrm{~V}$ to turn on, while the other drives the other two NMOS switches to $V_{C M B U F}$ to turn off. The common-mode buffer also drives the backgates of the NMOS switches to avoid the body effect. With a fixed $0.8 \mathrm{~V}$ NMOS threshold voltage, the overdrive voltage and the clock swing are kept constant at $0.8 \mathrm{~V}$ and $1.6 \mathrm{~V}$, respectively, with the changes in the input

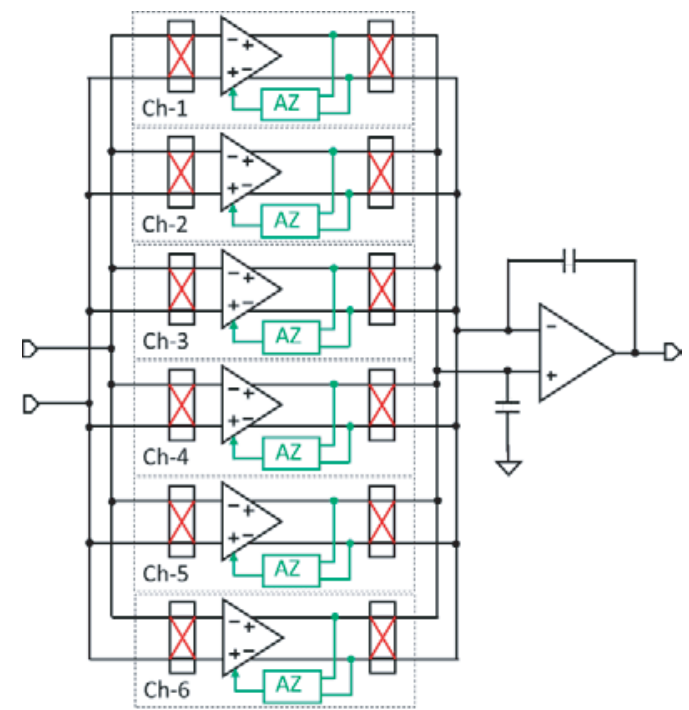

Fig. 12 Auto-zero and chopper op-amp with split six input channels

common-mode and supply voltages. This avoids the use of oversized switches, and improves the transient glitch, the residual offset, the CMRR, and the PSRR. The on-resistance of each switch is designed to be $200 \Omega$, so that the noise PSD of the overall op-amp is still dominated by the thermal noise of $G_{m l}$. With the combination of $5.6 \mathrm{nV} / \sqrt{\mathrm{Hz}}$ noise PSD, this op-amp achieves $0.5 \mu \mathrm{V}$ maximum offset and $400 \mathrm{pA}$ maximum input bias current which would be several times larger if conventional CMOS switches were used at the input.

\subsection{A Multi-Channel Auto-Zero and Chopper Op-Amp with Interleaved Clocks}

Another way to reduce the glitch amplitude is to spread out the energy of charge injection over time, by splitting input switches into multi-channels. In 2015, Kusuda proposed an auto-zero and chopper op-amp consisting of six input channels as shown in Fig. 12 [9]. Each channel consists of an input chopper, input transconductor, an output chopper, and an auto-zeroing loop to reduce the up-modulated ripple amplitude. As shown in the clock timing diagram in Fig. 13, each channel goes through auto-zeroing phase (AZ), chopping phase $(\mathrm{CH})$, and inverting-chop phase $\left(\mathrm{CH}_{\mathrm{INV}}\right)$. The phases of the six channels are interleaved, so that while two channels are in the $\mathrm{AZ}$ phase, the other four channels are either the $\mathrm{CH}$ or $\mathrm{CH}_{\mathrm{INV}}$ phases at a time. By always having four channels in the signal path, the required size of the switch and the required amount of transconductance are reduced by a factor of four.

Figure 13 also shows the expected glitch waveforms from each channel and their total combined. Compared to a chopper op-amp with single channel design, the glitch amplitude from each channel is four times smaller, and two channels generate glitches at a time. Therefore, the total glitch amplitude is expected to be two times smaller. Moreover, compared to ping-pong auto-zero and chopper op-amp 


\begin{tabular}{|c|c|c|c|c|c|}
\hline \multicolumn{6}{|c|}{ CLK Phase } \\
\hline Ch-1 & \multicolumn{2}{|c|}{$\mathrm{AZ}$} & \multicolumn{2}{|l|}{$\mathrm{CH}$} & $\mathrm{CH}_{\text {INV }}$ \\
\hline Ch-2 & $\mathrm{CH}_{\mathrm{INV}}$ & $A Z$ & & $\mathrm{CH}$ & \begin{tabular}{l|l} 
& $\mathrm{CH}_{\mathrm{INV}}$ \\
\end{tabular} \\
\hline Ch-3 & \multicolumn{2}{|c|}{$\mathrm{CH}_{\text {INV }}$} & \multicolumn{2}{|l|}{$A Z$} & $\mathrm{CH}$ \\
\hline Ch-4 & $\mathrm{CH}$ & $\mathrm{CH}_{\text {INV }}$ & & $A Z$ & $\mathrm{CH}$ \\
\hline Ch-5 & \multicolumn{2}{|c|}{$\mathrm{CH}$} & $\mathrm{CH}_{\text {INV }}$ & \multicolumn{2}{|r|}{$A Z$} \\
\hline Ch-6 & $A Z$ & $\mathrm{CH}$ & & $\mathrm{CH}_{\mathrm{INV}}$ & $A Z$ \\
\hline
\end{tabular}

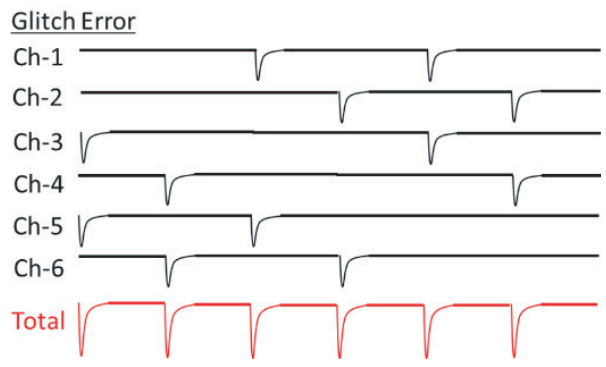

Fig. 13 The clock timing diagram and expected output glitch of Fig. 12

shown in Fig. 6, the proposed op-amp consumes less relative current for auto-zeroing, and is therefore more power efficient.

It is possible to further increase the number of channels to reduce the glitch amplitude. However, this decreases the time available for auto-zeroing phase, which may increase the current consumed in the auto-zeroing stage to meet the settling requirement. This also increases extra die area required for spacing between the adjacent channels. This opamp achieved a $5 \mu \mathrm{V}$ maximum offset and a $5.8 \mathrm{nV} / \sqrt{\mathrm{Hz}}$ noise PSD while consuming $840 \mu \mathrm{A}$ of current.

\section{Input Bias Current and Residual Offset Reduction Techniques}

\subsection{Nested Chopping}

In 2000, Bakker proposed nested chopping employing two different chopping frequencies as shown in Fig. 14 (a) [10]. An inner set of input and output choppers $\mathrm{CH}_{\text {INinner }}$ and $\mathrm{CH}_{\text {OUTinner }}$ are driven by a high chopping frequency $f_{\text {CHhigh }}$. While an outer set of input and output choppers $\mathrm{CH}_{\text {INouter }}$ and $\mathrm{CH}_{\text {OUTouter }}$ are driven by a low chopping frequency $f_{\text {CHlow }}$. Figure 14 (b) shows the expected transient waveforms along with the clock timings. In this example, $f_{C H, h i g h}$ is set four times higher than $f_{C H, l o w}$, and only the glitches generated by the inner chopping are shown for simplicity. First charge injection mismatch in $\mathrm{CH}_{\text {INinner }}$ causes voltage glitches $V_{i n A l}$ at the input of amplifier $A_{l}$.

These glitches are down-modulated by $\mathrm{CH}_{\text {OUTinner }}$ and thus produce DC error $V_{\text {outinner }}$. Then, the outer chopper $\mathrm{CH}_{\text {OUTouter }}$ up-modulates $V_{\text {outinner }}$ again, so that an output Vout contains no residual offset. Similarly, the input bias currents caused by $\mathrm{CH}_{\text {INinner }}$ are up-modulated by $\mathrm{CH}_{\text {INouter }}$, resulting in no DC input bias currents at the overall input pins. Through the operation of two output choppers, the offset and $1 / f$ noise of $A_{l}$ are up-modulated to two frequencies $f_{\text {CHhigh }} \pm f_{\text {CHlow }}$. Therefore, $f_{\text {CHlow }}$ can be set even

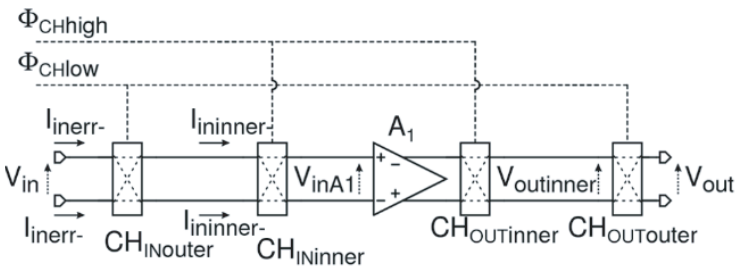

(a)

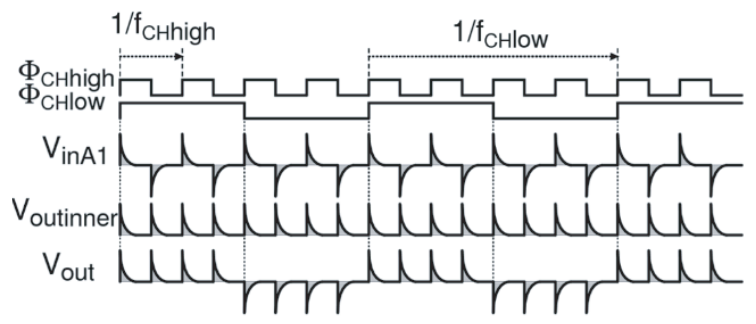

(b)

Fig. 14 Nested chopping: (a) circuit diagram; (b) transient waveforms.

below the $1 / f$ noise corner frequency, while almost eliminating the $1 / f$ noise from the baseband. Consequently, the residual offset caused by the outer chopper can be reduced to a very low level. In summary, the nested chopping technique utilizes two separate chopping frequencies, so that the $1 / f$ noise can be mitigated by a high $f_{C H h i g h}$ while the residual offset can be determined by a low $f_{C H l o w}$. With $f_{C H, l o w}=2 \mathrm{kHz}$ and $f_{C H, \text { high }}=16 \mathrm{kHz}$, the proposed op-amp achieves a $100 \mathrm{nV}$ offset and a $27 \mathrm{nV} / \sqrt{\mathrm{Hz}}$ noise PSD. One drawback is that the outer chopper creates spectral peaks at $f_{C H, l o w}$, which limits the usable signal bandwidth. Another drawback is that to achieve a targeted noise PSD, wider switches must be used, since two switches are connected in series.

\subsection{Input Bias Current Trimming by Compensating Charge Injection Mismatches}

In 2015, Kusuda proposed a charge mismatch compensation circuit as shown in Fig. 15 [9]. The four NMOS switches $\mathrm{S}_{1-4}$ are the input chopper switches and driven by positive and negative clock supplies $C V_{D D}$ and $C V_{S S}$ through inverters. Let us assume $S_{1}$ is associated with a coupling capacitor $C_{P}+\Delta C_{P}$, while each of the other three switches is associated with $C_{P}$. This capacitance mismatch $\Delta C_{P}$ causes a charge injection mismatch per clock period $1 / f_{C L K}$, generating initial input bias currents $I_{\text {binit }}$ and $I_{\text {binit }}$ equal to:

$$
\begin{aligned}
& I_{\text {binit }} \equiv I_{\text {binit }}=f_{C L K} \cdot \Delta C_{P} \cdot\left(C V_{D D}-C V_{S S}\right), \\
& I_{\text {binit- }} \equiv-I_{\text {binit }}=-f_{C L K} \cdot \Delta C_{P} \cdot\left(C V_{D D}-C V_{S S}\right) .
\end{aligned}
$$

To offset these error currents, the charge mismatch compensation circuit can inject charge mismatches with opposite polarity through four metal-metal coupling capacitors $C_{C 1-4}$. An adjustable positive supply $C V_{D D_{-} D A C P}$ drives $C_{C l}$ and $C_{C 3}$, while another adjustable positive supply $C V_{D D \perp D A C N}$ drives $C_{C 2}$ and $C_{C 4}$. By adjusting these supply voltages, the resulting compensation input bias currents $I_{b c o m p+}$ and 


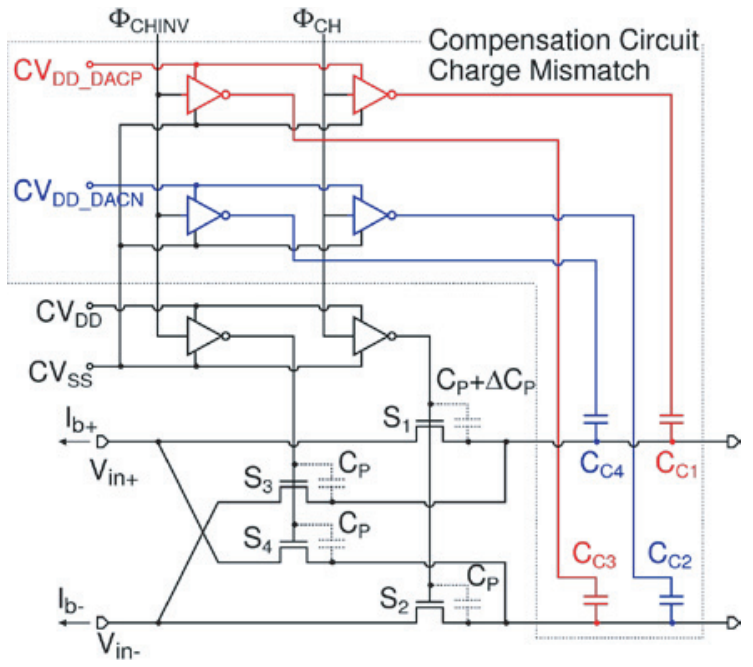

Fig. 15 The input chopping circuit and clock generator employing an adaptive clock boosting technique.

$I_{\text {bcomp- }}$ can be controlled to allow:

$$
\begin{aligned}
& I_{b c o m p+} \equiv I_{b c o m p} \\
& =2 f_{C L K} \cdot C_{C 1-4} \cdot\left(C V_{D D \_D A C P}-C V_{D D \_D A C N}\right), \\
& I_{b c o m p-} \equiv-I_{b c o m p} \\
& =-2 f_{C L K} \cdot C_{C 1-4} \cdot\left(C V_{D D \_D A C P}-C V_{D D \_D A C N}\right) .
\end{aligned}
$$

$C_{C 1-4}=2 \mathrm{fF}$ is only one-tenth of $C_{P}$, which will not significantly increase the absolute amount of charge injection nor, consequently, the magnitude of transient glitches. The resulting input bias currents $I_{b+}$ and $I_{b-}$ are given by:

$$
\begin{aligned}
& I_{b+}=I_{\text {binit }}+I_{b c o m p}, \\
& I_{b-}=-\left(I_{\text {binit }}+I_{b c o m p}\right) .
\end{aligned}
$$

Both $I_{b+}$ and $I_{b-}$ will be pushed toward zero by adjusting $I_{\text {bcomp }}=-I_{\text {binit }}$. Moreover, since the initial input currents and the compensation input bias currents are both proportional to the clock frequency, the cancellation remains stable with the change of the clock frequency over temperature.

The trimming is performed at a single temperature of $25^{\circ} \mathrm{C}$ - the initial input bias current is measured externally and then adjust $C V_{D D_{-} D A C P}$ and $C V_{D D_{-} D A C P}$ through an onchip DAC. This reduced the maximum input bias current from $1.5 \mathrm{nA}$ to $150 \mathrm{pA}$ at $25^{\circ} \mathrm{C}$. Changing the temperature to $85^{\circ} \mathrm{C}$ only increased the maximum input bias current up to $200 \mathrm{pA}$ without requiring additional trimming.

\section{Comparison of the Techniques}

Table 1 compares circuit techniques and the achieved specifications of chopper op-amps introduced in this paper, with the order of publication year (the oldest one is shown in the most left). Most chopper op-amp designs before 2011 ad-

\begin{tabular}{|c|c|c|c|c|c|c|}
\hline Author & $\begin{array}{c}\text { Bakker } \\
{[10]}\end{array}$ & $\begin{array}{c}\text { Tang } \\
{[6]}\end{array}$ & $\begin{array}{c}\text { Witte } \\
{[4]}\end{array}$ & $\begin{array}{c}\text { Burt } \\
{[5]}\end{array}$ & $\begin{array}{c}\text { Kusuda } \\
{[8]}\end{array}$ & $\begin{array}{c}\text { Kusuda } \\
\text { [9] }\end{array}$ \\
\hline Year & 2000 & 2002 & 2006 & 2006 & 2011 & 2015 \\
\hline$[\mu \mathrm{A}]$ & 100 & 800 & 700 & 17 & 1400 & 840 \\
\hline$[\mathrm{kHz}]$ & 16 & 15 & 32 & 125 & 200 & 1200 \\
\hline $\mathrm{Vos}, \max [\mu \mathrm{V}]$ & $* 0.1$ & 5.0 & 3.0 & 10.0 & 0.5 & 5.0 \\
\hline Ibias,max $[\mathrm{pA}]$ & **NS & 100 & $* * \mathrm{NS}$ & 200 & 400 & 150 \\
\hline GBW $[\mathrm{MHz}]$ & 0.4 & 2.5 & 1.37 & 0.35 & 4.0 & 3.1 \\
\hline $\mathrm{NSD}[\mathrm{nV} / \sqrt{\mathrm{Hz}}]$ & 19 & 20 & 15 & 55 & 5.6 & 5.8 \\
\hline NEF & 7.3 & 21.8 & 15.3 & 8.7 & 8.1 & 6.5 \\
\hline GBW/Isy & 4.0 & 3.1 & 2.0 & 20.6 & 2.9 & 3.7 \\
\hline Die area $\left[\mathrm{mm}^{\wedge} 2\right]$ & 1.5 & 0.67 & 3.6 & 0.7 & 1.26 & 1.58 \\
\hline $\begin{array}{l}\text { Ripple reduction } \\
\text { technique }\end{array}$ & - & $\begin{array}{l}\text { Auto } \\
\text {-zero }\end{array}$ & $\begin{array}{r}\text { Active } \\
\text { Filter } \\
\end{array}$ & $\begin{array}{l}\text { SC- } \\
\mathrm{NF}\end{array}$ & ACFB & $\begin{array}{l}\text { 6-channel } \\
\text { Auto-zero } \\
\end{array}$ \\
\hline $\begin{array}{l}\text { Glitch reduction } \\
\text { technique }\end{array}$ & - & - & - & - & $\begin{array}{r}\text { CLK } \\
\text { booster }\end{array}$ & $\begin{array}{r}{ }^{* * *} \text { CLK } \\
\text { interleave }\end{array}$ \\
\hline $\begin{array}{l}\text { Residual offset } \\
\text { reduction }\end{array}$ & $\begin{array}{r}\text { Nested } \\
\text { chopper }\end{array}$ & - & - & - & $\begin{array}{r}\text { CLK } \\
\text { booster }\end{array}$ & $\begin{array}{r}{ }^{* * *} \text { Charge } \\
\text { Trim }\end{array}$ \\
\hline
\end{tabular}
dressed ripple reductions. Among three designs [4]-[6], the one in [6] employs feedback to reduce initial offset, and thus can expect the lowest output ripple amplitude. However, this
Table 1 Comparison of specifications and circuit techniques of chopper op-amps.

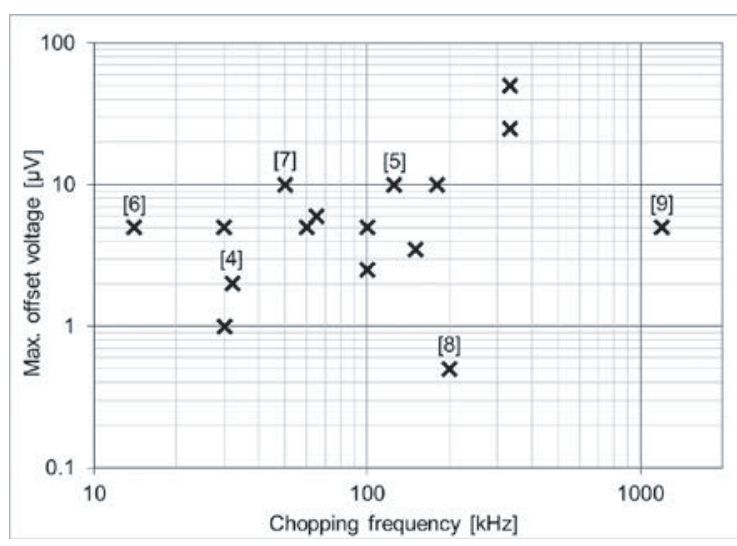

Fig. 16 Maximum offset versus chopping frequency of chopper opamps.

design consumes extra current for auto-zeroing, and therefore shows the highest noise efficiency factor (NEF), which indicates the amount of noise PSD when every design is scaled to the same current consumption [11].

Later than 2011, the two designs in [8], [9] employ feedback to better reduce resulting ripple, but also in power efficient ways to achieve lower NEF than the design in [6]. Moreover, those two designs employ techniques to reduce residual offset, and consequently, achieved competitive maximum offset Vos,max and maximum input bias current Ibias, max with relatively high chopping frequencies $\mathrm{fCH}$.

Figure 16 plots the chopping frequency versus the maximum offsets of 16 different op-amp designs. The offset tends to increase with the chopping frequency. The designs in [8] and [9] achieve relatively low offset of $0.5 \mu \mathrm{V}$ and $5 \mu \mathrm{V}$ with $200 \mathrm{kHz}$ and $1.2 \mathrm{MHz}$ of chopping frequency, respectively.

Figure 17 (a) shows the measured noise PSD of [8] and [9] to compare their glitch reduction techniques. Additionally, Fig. 17 (b) shows the corresponding integrated RMS noise over the frequencies. The op-amp in [9] shows smaller peak noise PSDs at its chopping and harmonic frequencies, thanks to the glitch and ripple reduction techniques. 


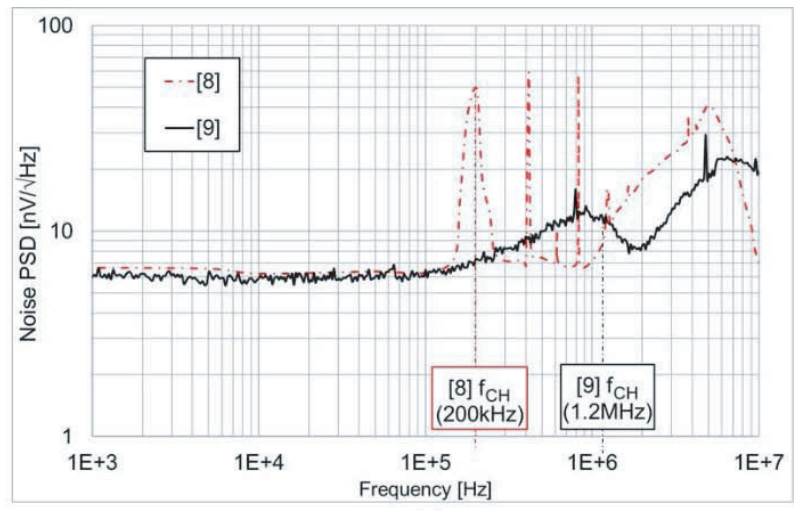

(a)

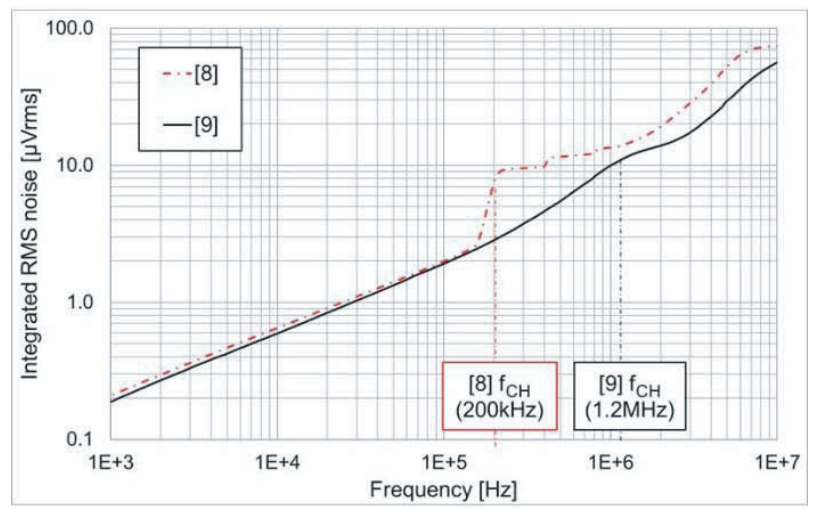

(b)

Fig. 17 (a) Noise PSD of [8] and [9]; (b) their integrated RMS noise

Consequently, for a $500 \mathrm{kHz}$ bandwidth, the integrated noise of the op-amps in [8] and [9] are $11.6 \mu \mathrm{V}_{\mathrm{rms}}$ and $5.4 \mu \mathrm{V}_{\mathrm{rms}}$, respectively. From another perspective, to target $10 \mu \mathrm{V}_{\mathrm{rms}}$ integrated noise, the usable signal bandwidths of the op-amps in [8] and [9] are $400 \mathrm{kHz}$ and $1 \mathrm{MHz}$, respectively.

\section{Conclusion}

This paper reviewed circuit techniques to reduce undesired switching artifacts - ripple, transient glitches, as well as residual offset and input bias current. As demonstrated by newer designs employing advanced circuit techniques [8], [9], noise spectra peaks due to switching artifacts can be reduced and pushed toward higher frequencies without significantly increasing DC errors. Such newer chopper op-amp designs can relax the requirements of post low-pass filters, and therefore serve a broader range of applications requiring wider usable signal bandwidth.

\section{References}

[1] C.C. Enz and G.C. Temes, "Circuit Techniques for Reducing the Effect of Op-Amp Imperfections: Autozeroing, Correlated Double Sampling, and Chopper Stabilization," Proc. IEEE, vol.84, no.9, pp.1320-1324, Sept. 1996.

[2] Y. Kusuda, "Reducing Switching Artifacts in Chopper Amplifiers," Ph.D. Thesis, Delft University of Technology, the Netherlands, May 2018.
[3] Q. Fan, J. Huijsing, and K. Makinwa, "Input Characteristics of a Chopped Multi-Path Current Feedback Instrumentation Amplifier," Proc. 4th Int. Workshop on Advances in Sensors and Interfaces, IWASI, pp.61-66, June 2011.

[4] J.F. Witte, K.A.A. Makinwa, and J.H. Huijsing, "A CMOS Chopper Offset-Stabilized Opamp," IEEE J. Solid-State Circuits, vol.42, no.7, pp.1529-1535, July 2007.

[5] R. Burt and J. Zhang, "A Micropower Chopper-Stabilized Operational Amplifier using a SC Notch Filter with Synchronous Integration inside the Continuous-Time Signal Path," IEEE J. Solid-State Circuits, vol.41, no.12, pp.2729-2736, Dec. 2006.

[6] A.T.K. Tang, "A $3 \mu$ V-Offset Operational Amplifier with $20 \mathrm{nV} / \sqrt{\mathrm{Hz}}$ Input Noise PSD at DC Employing both Chopping and Autozeroing," IEEE ISSCC Dig. Tech. Papers, pp.362-387, Feb. 2002.

[7] Y. Kusuda, "Auto Correction Feedback for Ripple Suppression in a Chopper Amplifier," IEEE J. Solid-State Circuits, vol.45, no.8, pp.1436-1445, Aug. 2010.

[8] Y. Kusuda, "A $5.9 \mathrm{nV} / \sqrt{\mathrm{Hz}}$ Chopper Operational Amplifier with $0.78 \mu \mathrm{V}$ Maximum Offset and $28.3 \mathrm{nV} /{ }^{\circ} \mathrm{C}$ Offset Drift," IEEE ISSCC Dig. Tech. Papers, pp.242-243, Feb. 2011.

[9] Y. Kusuda, "A $60 \mathrm{~V}$ Auto-Zero and Chopper Operational Amplifier With $800 \mathrm{kHz}$ Interleaved Clocks and Input Bias Current Trimming," IEEE J. Solid-State Circuits, vol.50, no.12, pp.2804-2813, Dec. 2015.

[10] A. Bakker, K. Thiele, and J.H. Huijsing, "A CMOS Nested-Chopper Instrumentation Amplifier with 100-nV Offset," IEEE J. Solid-State Circuits, vol.35, no.12, pp.1877-1883, Dec. 2000.

[11] M.S.J. Steyaert, W.M.C. Sansen, and C. Zhongyuan, "A Micropower Low-Noise Monolithic Instrumentation Amplifier For Medical Purposes," IEEE J. Solid-State Circuits, vol.22, no.6, pp.1163-1168, Dec. 1987.

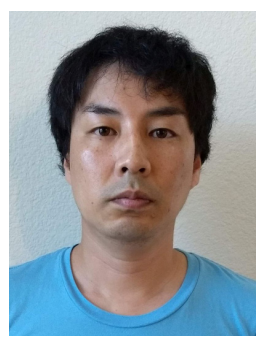

Yoshinori Kusuda joined Analog Devices Japan Design Center in 2004, after graduating from Tokyo Institute of Technology with an M.S. degree in Physical Electronics. Since then, he has been with Analog Devices and is currently based in San Jose, CA, U.S.A., working for precision CMOS analog designs, including stand-alone amplifiers and application specific mixed-signal products. In 2015 through 2018, he had been registered as a guest in the Electronic Instrumentation Laboratory of the TU Delft and obtained his Ph.D. degree. 